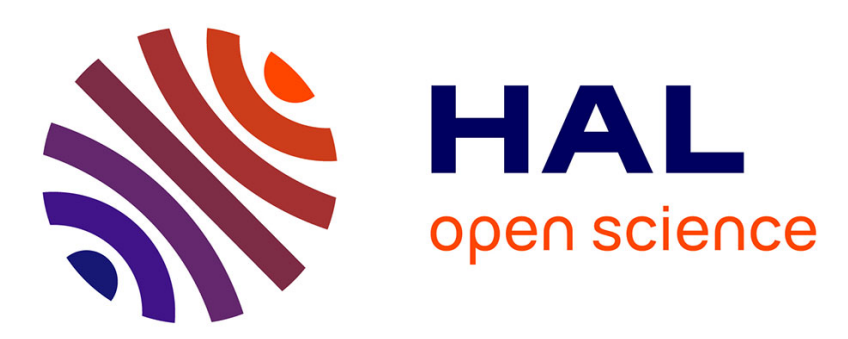

\title{
Gene dosage effects and transcriptional regulation of early mammalian adrenal cortex development
}

\author{
Pierre Val, \& Amanda Swain
}

\section{To cite this version:}

Pierre Val, \& Amanda Swain. Gene dosage effects and transcriptional regulation of early mammalian adrenal cortex development. Molecular and Cellular Endocrinology, 2010, 323 (1), pp.105. 10.1016/j.mce.2009.12.010 . hal-00593434

\section{HAL Id: hal-00593434 \\ https://hal.science/hal-00593434}

Submitted on 16 May 2011

HAL is a multi-disciplinary open access archive for the deposit and dissemination of scientific research documents, whether they are published or not. The documents may come from teaching and research institutions in France or abroad, or from public or private research centers.
L'archive ouverte pluridisciplinaire HAL, est destinée au dépôt et à la diffusion de documents scientifiques de niveau recherche, publiés ou non, émanant des établissements d'enseignement et de recherche français ou étrangers, des laboratoires publics ou privés. 


\section{Accepted Manuscript}

Title: Gene dosage effects and transcriptional regulation of early mammalian adrenal cortex development

Authors: Pierre VAL, \& Amanda Swain

PII: $\quad$ S0303-7207(09)00618-2

DOI: $\quad$ doi:10.1016/j.mce.2009.12.010

Reference: $\quad$ MCE 7391

To appear in: $\quad$ Molecular and Cellular Endocrinology

Please cite this article as: VAL, P., Swain, A., Gene dosage effects and transcriptional regulation of early mammalian adrenal cortex development, Molecular and Cellular Endocrinology (2008), doi:10.1016/j.mce.2009.12.010

This is a PDF file of an unedited manuscript that has been accepted for publication. As a service to our customers we are providing this early version of the manuscript. The manuscript will undergo copyediting, typesetting, and review of the resulting proof before it is published in its final form. Please note that during the production process errors may be discovered which could affect the content, and all legal disclaimers that apply to the journal pertain. 


\title{
Gene dosage effects and transcriptional regulation of early mammalian adrenal cortex development
}

\author{
Pierre VAL ${ }^{1}$ \& Amanda Swain ${ }^{2 *}$
}

${ }^{1}$ Centre National de la Recherche Scientifique, Unité mixte de Recherche 6247, Génétique, Reproduction et Développement, Clermont Université, 63177 Aubière, France ; ${ }^{2}$ The Institute of Cancer Research, Section of Gene Function and Regulation, 237 Fulham Road, London SW3 6JB, United-Kingdom. * Corresponding Author

\section{Overview of adrenal development}

The adrenal gland is an essential organ that controls stress response and participates in the control of blood pressure, lipid and glucose homeostasis and inflammation. It is made up of two compartments, an outer cortex and an inner medulla. Both secrete different essential hormones and defects in the function of the cortex are life-threatening. Medullar cells are derived from neural crest and produce catecholamines making them part of the sympathetic system. The adult cortex is divided in three zones, named from outer to inner: the zonae glomerulosa, fasciculata and reticularis. All zones have steroidogenic activity and produce mineralocorticoids (glomerulosa), glucocorticoids (fasciculata/reticularis) and androgens (in primates, but not rodents) [1]. Mineralocorticoid secretion is controlled by angiotensin II and glucocorticoids are produced in response to pituitary ACTH. Adrenal cortex development is first observed in the embryo at 8 weeks of gestation in human and at embryonic day 10.5 (E10.5) in mouse. Neural crest cells migrate to the area where the adrenal cortex is developing by the $9^{\text {th }}$ week of human gestation and at E13.0 in mouse. The developing adrenal then undergoes a series of histological changes and differentiation events to form a functional zonated adrenal gland in the adult. During human embryonic development, most of the adrenal cortex is composed of foetal adrenal cells surrounded by definitive adrenal cells. Foetal adrenal cells produce DHEAS, a steroid with androgenic properties, whereas definitive cells produce glucocorticoids. The foetal zone regresses by apoptosis after birth and has completely disappeared by the $6^{\text {th }}$ month of life. However, full functional and histological zonation of the definitive adrenal cortex is only observed around puberty. Until recently, the existence of a foetal and an adult adrenal cell population in mouse was controversial. The mouse X-zone was proposed to represent an equivalent of the human foetal adrenal due to its localisation at the cortex/medulla boundary, similar ultrastructural features and its transient nature. However, in contrast to the human foetal adrenal, the mouse X-zone was only apparent as a clearly defined structure at around 10-14 days post partum (dpp) and degenerated at puberty, in males, and after the first pregnancy in females [2]. Elegant lineage tracing studies in mouse have clearly shown that the X-zone is derived from foetal adrenal cells. These studies also showed that presumptive definitive adrenal cells were present in the mouse adrenal cortex during embryonic development [3,4]. Functional zonation in the mouse, characterized by the expression of specific steroidogenic enzymes in different areas of the cortex (e.g. Cyp11b1 in the zona fasciculata and Cyp11b2 in the zona glomerulosa) occurs in the perinatal period (Fig1). Ontogeny of the adrenal cortex has been covered extensively in various reviews [5-8]. The present review will focus on the early events of adrenal development with emphasis on formation and maintenance of the adreno-gonadal primordium, foetal adrenal cell fate determination and the emerging role of signalling molecules in the control of proliferation, differentiation and shaping of the cortex. 


\section{I- The Adreno-Gonadal Primordium (AGP)}

In mammals, the major steroid producing organs are the gonads and the adrenal cortex. Enzymes involved in the first steps of steroidogenesis are common to both gonads and adrenals (Cyp11a1 and 3 $\beta$-HSD) but the final steps are catalyzed by organ specific enzymes: Cyp21, Cyp11b1 and Cyp11b2 in the adrenal; Cyp19, 17 $\beta$-HSD and Cyp17 in the gonads, although the latter is expressed in primates' adrenals. Even though the specificity of expression is relatively strict in adult tissues, numerous reports have shown that some gonadspecific enzymes are expressed in the adrenal during development. Conversely, adrenal enzymes were observed in the developing gonad [9-14]. This suggests that, in agreement with histological studies, the adrenal cortex and gonads have a common embryological origin. Consistent with this, mice deficient for steroidogenic factor 1 (Sf-1), an essential regulator of steroidogenesis $[15,16]$, lack both adrenals and gonads [17, 18]. Detailed analysis of Sf-1 expression in rat embryos identified a group of positive cells that form the presumed adrenogonadal primordium or AGP, the shared precursor of both gonads and adrenals. These studies showed symmetrical lines of Sf-1-positive cells extending from the dorsal aorta to the dorsal coelomic epithelium of the E11.5 rat urogenital ridges. At E12.5, the adrenal primordium separated from the gonadal primordium on the dorsal aortal side, in the rostral area of the AGP [9]. In mouse, Sf-1 was detected as early as E9.0. The process of AGP development was similar and the adrenal anlage separated from the bipotential precursor at E10.5 (10-11 tail somites) $[19,20]$ (Fig1).

Several genes have been implicated in the development of the AGP. Mice lacking these factors have defects in both gonad and adrenal development.

\section{Steroidogenic Factor 1:}

Steroidogenic Factor 1 / Adrenal 4 Binding Protein (Sf-1/Ad4BP) was initially identified as the transcription factor binding to the Ad4 sequence found in the promoters of genes encoding several steroidogenic enzymes. It belongs to the nuclear hormone receptor super-family and has been shown to act as a master regulator of steroidogenic cell differentiation and function as well as of male sexual differentiation [15]. Consistent with the central role of Sf-1 in steroidogenic tissue differentiation, stable transfection of ES cells or adipose tissue-derived and bone marrow-derived mesenchymal cells with Sf-1 induces their differentiation to a steroidogenic fate [21-23]. Mice lacking Sf-1 show early adrenal and gonadal development, however, the two anlagen regress by apoptosis between E11.5 and E12.5 [17, 18]. This indicates that Sf-1 is essential for early development and maintenance of adrenal and gonadal primordia. Although there is increasing evidence that Sf-1 regulates proliferation in the adrenal primordium and adult adrenal, the mechanisms involved in apoptosis of the primordia in $S f-1-/-$ mice are not known [24-26]. SF-1 has recently been shown to repress expression of $\mathrm{NOV} / \mathrm{CCN} 3$, a factor with pro-apoptotic activity in H295R human adrenal carcinoma cell lines. Interestingly, NOV is expressed in foetal adrenal cells [27], suggesting that it may be involved in the apoptotic phenotype in Sf-1 deficient mice.

\section{Wilm's Tumour 1}

The Wilm's Tumour suppressor gene (WT1) was initially identified as a gene that was mutated in a subset of childhood kidney tumours. Patients with mutations in WT1 also showed various urogenital abnormalities that in some extreme cases led to male to female sex reversal. The WT1 gene encodes at least 24 different isoforms of a zinc finger containing protein through use of alternative promoters, alternative splicing and RNA editing. WT1 can bind DNA through its four zinc fingers DNA binding domain, but can also associate with 
spliceosomes in the presence of a three amino-acid motif (KTS) between the third and fourth zinc fingers [28, 29]. In mouse urogenital ridges, $W t 1$ is expressed as early as E9.5. Mice deficient for $W t l$ lacked kidneys and although a thickening of the early urogenital ridge was observed, this did not give rise to gonadal or adrenal development [30]. Interestingly, mice lacking either the +KTS or the -KTS isoform of Wt1 develop an adrenal, suggesting that the two isoforms can compensate for each other in adrenal development. However, mice mutant for the -KTS isoform show an increase in apoptosis at E11.5 in the anterior region of the gonad, which is reminiscent of $S f-1$ knockout mice [31]. This and the early urogenital phenotype of Wt1 deficient mice suggested that Wt1 acted upstream of Sf-1 in the genetic cascade leading to AGP development. Consistent with this, Wilhelm and Englert showed that Sf-1 expression was completely abrogated as early as E9.5 in Wt1-/- urogenital ridges, although expression of $L h x 9$, a gonadal marker, was unaltered. Using transgenic mice, they showed that Wt1 was directly activating transcription of Sf-1 through four Wt1 responsive elements found in a 674 bp promoter fragment that recapitulated Sf-1 expression in the urogenital ridges [32]. We have recently demonstrated that $\mathrm{Wt} 1$, in association with Cited2, plays an essential role in modulating Sf-1 dosage and triggering adrenal cortex differentiation ([20], see chapter II).

Odd-Skipped related 1:

Odd-Skipped related 1 is a presumptive transcription factor with three zinc fingers that has homology with the odd-skipped family of developmental genes in drosophila [33]. It is expressed in intermediate mesoderm and precedes $\mathrm{Wt1}$ expression in the urogenital ridges. Odd1 deficient mice have urogenital phenotypes similar to Wt1 mutant mice with absence of kidney development, blunted genital ridge thickening and no overt adrenal and gonadal differentiation. Interestingly, Wt1 expression is down-regulated in the E9.5 urogenital ridges of Odd1-/- embryos [34]. This suggests that Odd-1 acts upstream of Wt1 and controls its expression in intermediate mesoderm derivatives. Consistent with this, Odd 1 deficient mice have a more severe kidney phenotype (absence of metanephric mesenchyme condensation) than Wt1 mutant mice.

\section{II- Adrenal fate determination:}

At around E10.5 in the mouse, the bipotent AGP divides into the adrenal cortex anlage and the sexually undetermined gonad. The adrenal primordium progressively separates from the gonad in the rostral region. Analysis of genetically modified mice has identified two factors, Lxh9 and Emx2, as being important in specifying gonad differentiation. Mice mutant for either gene show adrenal development. However, gonad development is markedly impaired $[35,36]$. This indicates that these factors are specifically required to trigger gonad as opposed to adrenal development, although the underlying molecular mechanisms have not been identified. Until recently, factors involved in the determination of adrenal fate from the AGP were unknown.

\section{Cited2, Wt1 and Sf-1 dosage:}

Cited2 is a transcription cofactor devoid of DNA binding activity. It can act as a coactivator for transcription factors such as Lhx2, Tfap2 and the Ppar nuclear receptors by recruiting $\mathrm{Cbp} / \mathrm{p} 300$ to the promoter. It has also been shown to act as a corepressor for Hif1 $\alpha$ by competing for binding to $\mathrm{Cbp} / \mathrm{p} 300$. Mice lacking Cited2 were shown to lack adrenal glands at E17.5 although their gonads were apparently normal. These features were part of a more complex phenotype including neural crest migration defects, neural tube closure defects, abnormal body patterning and defective heart development [37-43]. We recently conducted a 
study of urogenital development in Cited 2 deficient mice. We showed that Cited 2 was expressed at high levels in the AGP and adrenal anlage and at lower levels in the overtly differentiated gonad. Consistent with this expression pattern, adrenal cortical development was not initiated in Cited 2 homozygous mutants. We demonstrated that this essential role of Cited 2 was dependent on its physical and functional interaction with Wt1 in the AGP, prior to adrenal formation. This interaction resulted in increased $S f-1$ promoter activity and $S f-1$ expression [20] (Fig2). Other studies have shown that modulations of Sf-1 dosage can affect gonadal and adrenal development to different degrees. In E13.5 Sf-1 heterozygous embryos, adrenal size was decreased by twelve fold compared to wild-type [25]. Increased proliferation later in development and augmented steroidogenic capacity per cell allowed partial recovery but adrenal responsiveness to stress was still markedly impaired in adults [44, 45]. In contrast, although early male gonad differentiation was affected in $S f-1+/-$ embryos, the adult gonad was fully functional [46]. This suggested that adrenal development in mouse was more sensitive to decreased Sf-1 dosage than gonadal development. Consistent with this, forced transgenic expression of Sf-1 in Sf-1 mutant mice rescued gonad development but not adrenal development, even though the transgene was expressed in both tissues in control animals [47]. In our experiments, $S f-1$ expression levels in the E10.5 AGP correlated with the severity of the adrenal phenotype. With $36 \%$ of wild-type dosage, as seen in Cited2-/- embryos, adrenal development was not initiated, whereas with $45 \%$ of wild-type levels, as seen in Cited2+/$W t 1+/$ - embryos, adrenal development was initiated but adrenal growth was markedly impaired. Therefore we proposed that Sf-1 dosage had to reach a critical threshold, at a particular stage, to trigger adrenal development from the AGP and that Cited2, together with Wt1, were required to increase Sf-1 expression above that threshold (Fig2) [20]. It is intriguing that $\mathrm{Wt} 1$, which is not found in the overtly differentiated adrenal $[20,48]$, plays an essential role for its determination from the AGP. These data are confirmatory evidence of the existence of the AGP and the common origin of the gonad and adrenal cortex. Also consistent is the early gonad phenotype observed in Cited2-/- embryos, characterised by decreased expression of Cypllal and Sox9 in the male gonad at E11.5. Preliminary studies reveal that this defect can lead to male to female sex reversal in different genetic backgrounds (unpublished work).

\section{Sf-1 dosage, mouse versus human:}

Consistent with the phenotype in $S f-1$ homozygous mutant mice, patients with $S F-1$ mutations that were initially identified, were shown to have both adrenal insufficiency and gonadal dysgenesis [49, 50]. Recent reports have described patients with heterozygous nonsense mutations in $S F-1$ that result in premature translation termination and undetectable protein production from the mutant allele [51, 52]. These patients are thought to be true models of $S F-1$ haploinsufficiency in humans. Surprisingly, they show gonadal dysgenesis but normal adrenal function. Several heterozygous missense mutations have also been shown to cause gonadal dysgenesis despite normal adrenal function [53-56]. Although the latter observation may reflect impairment of tissue-specific cofactor recruitment, this suggests that in humans, as opposed to mouse, gonad development is more sensitive to reductions in SF-1 dosage than adrenal development.. Although this may seem incompatible with our model of adrenal differentiation, several considerations must be taken into account. One major difference between these studies is that human patients are assessed after birth when compensatory growth and differentiation events have masked some of the embryonic phenotype. Therefore dissimilar phenotypes might reflect a difference in compensation or overall adrenal functional robustness rather than a difference in the initial events of determination and differentiation. Another important point is the effect of the genetic background on the sensitivity of these subtle gene dosage systems. For instance, we have shown that the levels of $S f-1$ in AGP of 
mice with a mixed genetic background was 1.4 fold higher than in mice with a pure C57/B16 background [20]. Consistent with this, we observed signs of adrenal development in Cited 2 mutant embryos on a mixed genetic background (unpublished observations). It is thus conceivable that genetic modifiers are impinging upon the effects of SF-1 dosage in the human population, giving rise to different phenotypes in patients with similar mutations. Further analyses of adrenal development in $S f-1$ or Cited 2 mutant animals on different genetic backgrounds might uncover such modifiers.

It is now clear that $\mathrm{Sf}-1$ dosage is crucial for steroidogenic cell fate determination, differentiation and proliferation control (Fig3). One of the main challenges is now to define which Sf-1 target genes are involved in these processes and how Sf-1 dosage is transcriptionally interpreted in order to trigger expression of tissue-specific pathways.

\section{III- Regulation of Sf-1 dosage}

\section{Sf-1 locus: transcriptional enhancer elements}

Because of the dosage-sensitive nature of Sf-1 activity, its spatio-temporal expression has to be tightly controlled. Analyses in transgenic mice have allowed the identification of promoter fragments and enhancers that control expression of Sf-1 in different tissues.

\section{Adreno-gonadal primordium enhancer element}

Studies in transgenic mice with the green fluorescent protein reporter gene, have shown that fifty kilobases of $S f-1$ upstream regulatory regions and intragenic sequences (first exon and first intron) were sufficient to recapitulate endogenous Sf-1 expression pattern excepting in the anterior pituitary [57]. Within this genomic locus, $674 \mathrm{bp}$ of a sequence upstream of the transcriptional start site were sufficient to drive expression of a LacZ reporter gene to the AGP of transgenic mice [32]. Mutation analysis showed that this region harboured four Wt1 responsive elements that were essential for promoter activity in the AGP as well as responsiveness to Cited2 [20,32]. However, this region was not sufficient to drive expression in the adrenal after its overt differentiation from the AGP.

\section{Fetal Adrenal Enhancer (FAdE)}

Zubair and colleagues have recently identified an $S f-1$ regulatory element that is highly conserved between mouse and human. This $600 \mathrm{bp}$ enhancer fragment is contained in the fourth intron of $S f-1$ and was shown to drive specific reporter gene expression to the adrenal primordium of transgenic mice, as early as E10.5 [3]. Therefore, this DNA region was called Fetal Adrenal Enhancer or FAdE. Among the conserved transcription factor binding sites in FAdE were two Sf-1 responsive elements (two others were mouse-specific), one Pbx/Prep and one $\mathrm{Pbx} / \mathrm{Hox}$ binding site. All $\mathrm{Sf}-1$ responsive elements were functional in binding assays, suggesting that Sf-1 could auto-regulate its own expression. However, expression of the reporter was shown to persist in the E11.5 adrenal anlage when all four sites were mutated or when the wild-type transgene was assessed in Sf-1-/- mice. This indicated that Sf-1 was dispensable for the initiation of expression of its own fetal adrenal enhancer. It was nonetheless required at later stages of development as shown by the absence of expression of the mutated transgene in the E17.5 adrenal [3]. Interestingly, mice lacking Pbx1, a member of the TALE family of homeodomain transcription factors, lacked adrenals at E14.5, had rudimentary gonads (but no sex reversal) and showed renal abnormalities. Pbxl-/- embryos showed decreased expression of Sf-1 in the urogenital ridge at E10.0 although Wt1 expression was similar to wild-type levels. This suggested that Pbx1 was acting either at the same level or downstream of Wt1 in the genetic cascade controlling Sf-1 expression in the AGP. 
However, the marked decrease in proliferation observed in the E10.5 urogenital ridge of $\mathrm{Pbx}$ mutant embryos could also account for reduced expression of Sf-1 [58]. In order to address this question, Zubair and colleagues analysed the function of the Pbx/Prep and Pbx/Hox binding sites within the FAdE sequence. Using electrophoretic mobility shift assays, these sites were shown to allow cooperative binding of Hox, Pbx 1 and Prep factors. When one or the other site was mutated in the context of the FAdE sequence in transgenic mice, expression of the reporter gene was decreased in the adrenal anlage. When both sites were mutated, expression of the reporter was abrogated in the E11.5 adrenal primordium. Altogether, these observations indicated that Pbx1 was required to initiate Sf-1 enhancer activity in the foetal adrenal and that, at later stages, Sf-1 maintained FAdE activity by binding to its cognate responsive elements [3]. Interestingly, Sf-1 expression was not altered in $\mathrm{Pbxl}+/$ - adult adrenals. However, Sf-1 transfection in Y1 adrenocortical cells increased Pbx1 expression. Conversely, $\mathrm{Pbx} 1$ expression was decreased in $S f-1$ haploinsufficient animals. This indicated that $\mathrm{Sf}-1$ could stimulate Pbx1 expression in the adult adrenal [59]. Whether a similar feedforward loop exists in the foetal adrenal is still unknown.

The identification of sites that bind both Pbx1 and Hox factors within FAdE indicate that members of the Hox gene family are important in controlling Sf-1 levels in the adrenal. Hox proteins have been shown to control the patterning of different structures during embryonic development by regulating the expression of subsets of target genes [60, 61]. It is thus likely that Hox factors define the antero-posterior area where the adrenal anlage arises from the AGP. Cell sorting experiments with mice expressing GFP under the control of the FAdE sequence have identified Hoxb5, Hoxb9 and Hoxc5 as potential candidates in this process [3].

Altogether these results suggest a three-step model for the control of Sf-1 expression in adrenal precursors and the foetal adrenal: 1) Wt1 induces Sf-1 proximal promoter activity in cells of the AGP. Cited 2 increases Sf-1 expression through interaction with Wt1, triggering adrenal differentiation in a subset of cells 2) FAdE is activated in the defined adrenal cortex precursor cells through Pbx1 action, increasing Sf-1 production 3) Sf-1 maintains activity of its own enhancer by binding to its cognate sites. One question that remains open to investigation is the potential role of Cited 2 on FAdE activity. Also, the enhancer responsible for Sf-1 expression in the definitive adrenal cortex remains to be identified.

\section{Sf-1 dosage modulators}

M33

M33 is a mammalian member of the Polycomb Group proteins that have been shown to repress Hox gene transcription at the level of chromatin structure. M33 deficient mice have partial male-to-female sex reversal and smaller adrenals [62, 63]. Consistent with these phenotypes, gonad development is markedly impaired at E11.5 and expression of $S f-1$ is reduced to $50 \%$ of wild-type levels in the E16.5 adrenals. This suggests that M33 controls Sf1 expression in the AGP. Indeed, ChIP assays showed that M33 binds to Sf-1 upstream regulatory regions in mouse Y1 adrenocortical cells. These regions did not overlap with the 674bp AGP promoter or with the FAdE [63]. The role of M33 in the control of Sf-1 expression in the AGP or the adrenal primordium thus remains unclear, although it could be implicated in the formation of intergenic boundaries impinging upon expression of the whole locus.

\section{Podl}

Pod1 (or Tcf21) is a bHLH transcription factor that has been shown to play important roles in the development of numerous tissues [64]. It is expressed in the urogenital ridges as early as 
E9.5 and delineates the boundary between the gonad and mesonephros in both sexes at E11.5. At later stages Pod1 expression becomes sexually dimorphic and it is found in the testis interstitium but not within the ovary [65]. Pod1 was shown to repress Sf-1 expression in tissue culture experiments [65-67]. Consistent with this, Pod1 deficient embryos showed an expanded Sf-1 expression domain at the boundary between the gonad and mesonephros. The number of Sf-1 positive cells in the gonad of both sexes was also increased and gonad development and testis differentiation were affected in these mice. Although Pod 1 was not directly involved in adrenal development, its disruption prevented the physical separation between the gonad and adrenal primordia. Interestingly, there was no increase in adrenal markers expression in the gonad which suggested that differentiation and sorting of the precursors were not affected [66]. Thus Pod1 acts to restrict Sf-1 expression such that the adrenal and gonad primordia are separated during development. This suggests that the mechanism of appearance of the adrenal primordium from the AGP is one of expansion of the AGP that is then restricted to form the two anlagen. However, a model where AGP cells migrate to the region where the adrenal forms cannot be excluded with the available information.

\section{Daxl}

The Daxl gene encodes a putative transcription factor that belongs to the nuclear hormone receptor family although it is devoid of the classical zinc finger DNA binding domain (reviewed in [68]). DAX1 was initially identified as the gene present in the region of the $\mathrm{X}$ chromosome found duplicated in patients with male to female sex reversal. Mutations or deletions of regions of the $\mathrm{X}$ chromosome containing DAX1 in humans cause hypogonadotrophic hypogonadism and adrenal hypoplasia congenita, characterised by the persistence of the foetal adrenal zone [69]. Sf-1 and Dax1 are coexpressed in steroidogenic tissues and other areas early in development [70, 71]. In vitro studies have shown that Dax1 acts as a repressor of steroidogenesis and of Sf- 1 transcriptional activity by recruiting corepressors to Sf-1 target promoters (reviewed in $[68,72]$ ). However, recent observations show that Dax-1 can also function as a coactivator for Sf-1 by recruiting the RNA coactivator SRA and TIF2 [73]. Thus depending on the cellular context, Dax-1 has the potential to repress or stimulate Sf-1 transcriptional activity.

Deletion of the C-terminal domain of Dax 1 in mouse gives rise to a mild adrenal phenotype characterised by the persistence of a presumptive foetal adrenal cortex in adults, reminiscent of the human pathology, but no adrenal hypoplasia [74]. Studies with this mutant allele show partial rescue of histological and functional defects in $S f-1$ haploinsufficient mice, in agreement with the repressive activity of Dax1 on Sf-1-mediated gene transcription [45]. These observations suggest that Dax1 does not play a major role in early adrenal development. However, these mice do not represent a complete loss of Dax1 function as truncated Dax 1 protein may be produced from the mutant locus. It is likely that such a protein would retain some of Dax1 biological activity, which would affect the interpretation of the mutant phenotypes.

Recent lineage tracing experiments have suggested that Dax1 acts to restrict adrenal differentiation from the AGP. When the $S f-1$ foetal adrenal enhancer sequence (FAdE) was used to drive expression of CRE, recombination was not only observed within the adrenal but in a few cells in the anterior region of the gonad primordium at E11.5 [4]. These cells were not observed in transgenic mice where the same enhancer was used to drive LacZ expression [3]. This indicated that cells within the gonad had been induced to become adrenal precursors but that their differentiation was subsequently prevented. Interestingly, careful analysis of Dax 1 expression in the region showed that it was found in the gonad but not the adrenal compartment at E10.5. In vitro studies also showed that Dax1 could repress FAdE activity [4]. 
Therefore it is tempting to speculate that Dax 1 prevents adrenal differentiation in the cells of the AGP that are targeted to gonad fate. We previously described cells with adrenal markers expression -the so-called adrenal-like cells- within the testicular interstitium during embryonic development and after birth [14]. It would be interesting to investigate If Dax1 prevents adrenal differentiation in the AGP, by analysing the population of adrenal-like cells in the Dax1 mutant gonads of both sexes, which should be expanded. Thorough analysis of the early stages of adreno-gonadal development in Dax1 knockout mice may clarify this point.

\section{IV- Signalling molecules and adrenal development}

Various signalling pathways have been shown to be crucial for cell interactions that modulate patterning, differentiation and proliferation in numerous tissues of the embryo. Recent genetic studies in mouse have implicated members of the Wnt, Hedgehog and Fgf signalling pathways in the development of the adrenal cortex.

\section{Wnt/ $\beta$-catenin}

The Wnt or 'wingless-like MMTV integration site' family of morphogens signal through an evolutionarily conserved pathway that has been implicated in many processes in embryonic development, regeneration, stem cell maintenance and cancer. The canonical Wnt pathway modulates the posttranslational regulation of $\beta$-catenin, by controlling the activity of a multiprotein complex that targets $\beta$-catenin for ubiquitin dependent degradation via the proteasome. $\beta$-catenin is a multifunctional protein with one of its main functions being to act as a transcriptional cofactor for members of the Lef/Tcf family of transcription factors. In this capacity, $\beta$-catenin acts as a final downstream target of the action of the Wnt family of secreted signalling molecules (for an in depth review of this pathway read [75])

A role for $\beta$-catenin in adrenal development has recently been demonstrated by the study of mice with a conditional allele of this gene and a transgene containing Cre recombinase driven by $S f 1$ regulatory sequences [76]. In this study two transgenic Cre lines were used: one carried one copy of the transgene and had low recombination efficiency in the adrenal cortex $\left(\mathrm{Sf1} / \mathrm{Cre}^{\mathrm{Low}}\right)$; the other line carried five copies of the same transgene and displayed high recombination efficiency $\left(\mathrm{Sf} 1 / \mathrm{Cre}^{\mathrm{High}}\right)$. The $\mathrm{Sf}-1 / \mathrm{Cre}^{\mathrm{High}}$ transgene was shown to induce complete inactivation of $\beta$-catenin as early as E12.5. This resulted in a drastic decrease in proliferation and expression of Sf-1. Expression of the steroidogenic enzymes Cyp11a1 and $3 \beta \mathrm{HSD}$ was also markedly reduced. By E18.5, most of the adrenal gland (cortex and medulla) had disappeared in $\beta$-catenin deficient embryos. These observations showed that $\beta$-catenin was required to stimulate and/or maintain proliferation of adrenal cortical cells during embryonic development. In contrast, partial inactivation of $\beta$-catenin with the $\mathrm{Sf}-1 / \mathrm{Cre}{ }^{\text {Low }}$ transgene had no major effect on adrenal histology and differentiation until 30 weeks post partum. At this stage, a subset of mutant mice presented thinning and disorganisation of the adrenal cortex as well as increased apoptosis. These also showed decreased expression of Sf-1 and steroidogenic markers. At 45 weeks post partum, the thinning phenotype was evident in all $\beta$-catenin deficient mice. This suggested that renewal of adrenal cortical cells over time was markedly impaired in $\beta$-catenin deficient mice. $\beta$-catenin has been shown to play important roles in progenitor cell expansion and lineage decisions in multiple tissues [77]. In the adult adrenal, $\beta$-catenin was found to be expressed in the subcapsular area [76] which has been postulated to contain adrenal progenitor cells [78]. It is thus tempting to speculate that $\beta$ catenin plays a role in renewal and/or commitment of progenitors, in order to maintain adrenal cortical function throughout life. 
The impairment of proliferation in the embryonic adrenal cortex of $\beta$-catenin deficient mice is consistent with clinical observations suggesting that constitutive ectopic activation of $\beta$ catenin in adult adrenals could be involved in the pathogenesis of benign and malignant adrenal cortical tumours $[79,80]$. Analysis of $S f-1$ haplo-insufficient mice suggested that Sf-1 was involved in the control of proliferation in the adrenal cortex [25, 44, 81]. Sf-1 was overexpressed in certain types of adrenal tumours, suggesting that increased Sf- 1 dosage could participate to the tumorigenic process [82-85]. Consistent with this, when Sf-1 was overexpressed in transgenic mice, a two fold increase in Sf-1 dosage was sufficient to induce adrenal tumour development soon after birth. Tumours in these mice first developed by expansion of capsular/sub-capsular cells and presented a mixed adrenal and gonadal phenotype [26]. Interestingly, Sf-1 and $\beta$-catenin have been shown to co-ordinately control expression of multiple genes [86-88]. Furthermore, $\beta$-catenin and Lrh-1, a nuclear receptor closely related to $\mathrm{Sf}-1$ that binds to the same DNA response element [89], cooperate to stimulate expression of Cyclin D1 and Cyclin E1 in intestinal crypts [90, 91]. Therefore, it is likely that $\beta$-catenin and Sf-1 cooperate to control cell proliferation and progenitor cell commitment in the adrenal cortex.

The only Wnt molecule reported to be expressed in the adrenal is Wnt4, which is thought not to signal through the canonical pathway [10]. However, studies in the gonad showed that Wnt 4 can act through $\beta$-catenin as Wnt4 mutant embryos show a similar phenotype to $\beta$ catenin mutants in the developing ovary [92-94]. Wnt4 was shown to be expressed in the early adrenal and restricted to the outermost area of the cortex as early as E14.5. Analysis of the adrenal phenotype of Wnt4 mutant embryos showed a milder phenotype than the $\beta$ catenin mutants, in that adrenal development did occur but zona glomerulosa differentiation was defective with reduced Cyp11b2 expression and low aldosterone production [10]. This suggested that other $\beta$-catenin activating signalling molecules were present in the adrenal. A phenotype shared by Wnt4 and $\beta$-catenin mutant embryos was the presence of cells expressing the adrenal specific marker, Cyp21 within the anterior region of the ovary. Cell migration assays showed that these cells migrated from the adrenal area to the mutant gonad in Wnt4 mutant embryos $[10,94]$. This indicated that this pathway was required to prevent migration of adrenal cells to the gonad. Reciprocally, Wnt4 mutant male adrenals showed ectopic expression of Cyp17. This 'gonadal' steroidogenic enzyme is normally expressed in the female but not the male developing adrenal and is never found in the adrenal of rodents after birth [10]. Whether this observation reflects abnormal migration of gonadal cells to the adrenal or de-repression of the gonad differentiation programme in the adrenal is unknown.

\section{Sonic Hedgehog (Shh)}

The hedgehog signalling pathway is involved in essential processes such as patterning of tissues during development, cell proliferation and differentiation. Activity of the pathway relies on three ligands: Sonic Hedgehog (Shh), Indian Hedgehog (Ihh) and Desert Hedgehog (Dhh). These bind the Patched1 and Patched2 (Ptch1, Ptch2) membrane receptors on Hedgehog responding cells. Binding of Hedgehogs to their receptors triggers a complex signalling cascade that culminates with modulation of Gli transcription factor activity (For an in depth review of this pathway, read [95]).

Hedgehog signalling has been implicated in steroidogenic cell activation within the gonad. Yao and colleagues showed that in the developing testis, Dhh was expressed by Sertoli cells at E11.5 whereas Ptch1 was present in the interstitium at E12.5, suggesting that Hedgehog signalling could be involved in the development of Leydig cells. Consistent with this, genetic ablation of Dhh or treatment with the hedgehog signalling inhibitor cyclopamine, resulted in an impairment of Leydig cells differentiation, characterised by decreased expression of Sf-1 and Cyp11a1 [96]. Although an effect of hedgehog signalling on Leydig cells precursors 
recruitment could not be ruled out, this suggested that hedgehog signalling was required for up-regulation of Sf-1 expression (either direct or indirect) and subsequent Leydig cell differentiation.

The first hint that Hedgehog signalling may be involved in adrenal development came from the observation of adrenal hypoplasia in infants with Pallister-Hall syndrome (PHS, OMIM \#146510), an autosomal dominant disorder caused by truncations of GLI3 [97, 98]. Consistent with this, transgenic mouse models with a similar truncation of Gli3 also showed adrenal agenesis at late gestation [99]. Preliminary experiments in mouse embryos showed that Shh was the only member of the family to be expressed at the periphery of the cortex as early as E14.5 and in the subcapsular area after birth. Ptch1 was found in an adjacent external layer of cortical cells, suggesting that active hedgehog signalling occurred in adrenal areas that contain progenitor cells ([95, 100] and our unpublished observations). Consistent with this, preliminary observations by King and colleagues show that adrenal gland development is impaired as early as E12.5 in Shh deficient mice [95]. Whether this affects proliferation, differentiation, zonal patterning or renewal of the adrenal cortex has yet to be determined. The overlap of Shh and $\beta$-catenin expression in the subcapsular area suggests that the two pathways may interact to control progenitor cells renewal and commitment as observed in other development and cancer models [101-104]. Detailed genetic analysis of Hedgehog and $\beta$-catenin signalling interactions should provide insight into the role of these pathways in adrenal gland development and homeostasis.

Fibroblast Growth Factors (FGFs) and FGF-receptor type II (FGFR2)

FGFs constitute a vast family of signalling proteins (at least 28 members) involved in diverse processes such as cell differentiation, proliferation, angiogenesis, and wound healing. FGFs mediate their activities by binding to a family of four receptor tyrosine kinases (FGFR1-4). This triggers intra-cellular signalling through the MAP kinase (MAPK), the phosphatidyl inositol 3 kinase (PI3K) and protein kinase $\mathrm{C}$ (PKC) pathways (for review see [105]).

FGF9, through FGF-receptor type 2 is essential for male sex determination by controlling proliferation of pre-sertoli cells in the testis [106-108]. Interestingly, mice in which Fgfr2 was conditionally deleted using a $S f-1$-driven Cre line had barely detectable adrenal development at E15.5 [108]. The factor responsible for this effect on adrenal growth is unknown. However basic FGF (bFGF/FGF2) which was shown to stimulate proliferation in a variety of adrenal cell types is a likely candidate [109-112]. In adult adrenals, bFgf expression is stimulated by ACTH and it was proposed to mediate the mitogenic effects of ACTH [109, 110, 113]. Although ACTH is involved in adrenal growth during development [7], a potential impairment of ACTH mitogenicity owing to Fgfr2 deficiency is unlikely to account for adrenal agenesis in Fgfr2-/- embryos at E15.5. Indeed, although adrenals in mice deficient for POMC, the peptide precursor of ACTH, are hypoplastic at birth, their size and histology can be restored upon chronic ACTH treatment[114-116]. This indicates that embryonic adrenal development is only mildly impaired in the absence of ACTH. Therefore FGFs must have a role during early adrenal development beyond growth response to ACTH. Interestingly, in adult rat adrenals, bFgf production was detected in the zona glomerulosa and bFgf binding sites were found in the zona glomerulosa and in cells of the capsule [110, 111]. This subcapsular localisation is reminiscent of both $\beta$-catenin and Shh and suggests a role for Fgf signalling in the control of adrenal progenitor cells homeostasis.

The role of signalling pathways in adrenal development was overlooked for a long time. Recent analysis of the mutant mouse models presented in this chapter has emphasised the need for in-depth research in this area. Although the published data mainly points to a role of $\beta$-catenin, hedgehog and Fgf signalling in the stimulation of adrenal proliferation it is likely 
that these pathways also participate in the differentiation and zonal shaping of the adrenal cortex. Other signalling pathways may also be involved, for example the TGF $\beta /$ activin/BMP signalling pathway has been shown to prevent adrenal tumorigenesis and gonadal differentiation of adrenal progenitor cells $[117,118]$. Although this data relies on observations in adult mice, it is possible that this pathway could play a role during specification of the adrenal cortex at earlier stages.

\section{V- Conclusion}

Although the adrenal gland was first described by Eustachius in the $16^{\text {th }}$ century, our understanding of the molecular and cellular mechanisms required for its embryonic development is still limited. The identification of Sf-1 and the analysis of the phenotype of mice with a genetic ablation of this gene in 1994 undoubtedly enhanced our knowledge of how adrenal cortex development and physiology were regulated at the molecular level. More recent work has shown that Sf-1 dosage is of crucial importance to specify adrenal cell fate from the bipotent AGP and has identified some of the mechanisms required to ensure that the proper levels are available to ensure adrenal differentiation. Other than Sf-1, very little is known about what factors are required to determine the differentiation of the adrenal cortex. $\mathrm{Sf}-1$ target genes involved in this process and zone specific factors that interact with Sf-1 providing specificity have still not been identified. An in depth study of the different signalling pathways that have been shown to be required for adrenal cortex development will be essential not only in establishing the role of these factors but in identifying cellular interactions that are important in this process. 


\section{Figure legends}

Figure 1. Adrenal development in mouse embryos.

The key events of adrenal development are shown. Adrenal cortex and gonads are derived from the adreno-gonadal primordium (AGP, green cells), a common progenitor cell population. At E10-E10.5 foetal adrenal cortical cells progressively separate from the AGP in the rostral area (light blue cells). Remaining cells of the AGP form the bipotential gonad anlage (pink cells). This differentiates as a testis upon expression of Sry in the male embryo or as an ovary in the female embryo. After separation from the AGP, the adrenal anlage is encapsulated and colonised by neural crest cells that will form the medulla. Lineage tracing experiments have shown that foetal adrenal cells derived from the AGP give rise to definitive adrenal cells (dark blue cells) that will form the adult cortex. The first signs of functional adrenocortical zonation appear at around E16 but it is not until the third week post partum that all four cortical zones (glomerulosa, fasciculata, reticularis, X-zone) are clearly defined. The $\mathrm{X}$-zone is thought to represent a remnant of the foetal adrenal cortex. It regresses after the first pregnancy in females or at puberty in males. Factors involved in AGP development as well as adrenal and gonadal fate determination are shown. See text for a detailed description of their function. Abbreviations: E, Embryonic day; dpp, days post partum.

Figure 2. Effect of Cited2 on Sf-1 dosage and adrenal fate determination in the AGP. Molecular and genetic analysis of Cited 2 mutant mice has shown that this cofactor interacts with Wt1 in order to modulate Sf-1 dosage in the AGP, before overt adrenal differentiation. In wild-type mouse AGP, Cited2 and Wt1 stimulate Sf-1 expression above the threshold required to trigger adrenal development. In Cited2 deficient mice, Sf-1 expression is decreased to $36 \%$ of wild-type levels. This level is sufficient to trigger development of the gonad although its later differentiation is impaired. However, Sf-1 dosage does not reach the threshold required to trigger adrenal formation. Adrenal primordium development from the AGP does not occur. Pictures show RNA in situ hybridisation for $S f-1$ in wild-type (left panel) or Cited2-/- embryos (right panel). Abbreviations: AGP, adreno-gonadal primordium; Ad, adrenal primordium; Go, gonad primordium. Pictures were reproduced with the kind permission of The Company of Biologists Ltd. They originate from Val et al., Development 134(12):2349-2358.

Figure 3. Factors controlling Sf-1 dosage in early embryonic development.

Factors that stimulate Sf-1 expression in the AGP (Wt1 and Cited2) through the 'AGP enhancer' or in the foetal adrenal (Pbx1/Hox) through the foetal adrenal enhancer (FAdE) are shown on the left (red arrows). Whether Cited 2 cooperates with Pbx1 or other factors to stimulate FAdE activity is not known. How M33 stimulates Sf-1 expression is yet unclear. Factors that decrease Sf-1 dosage either at the transcription level (Pod1) or by inhibitory protein-protein interactions (Dax1) are shown on the right (blue arrows). Increased Sf-1 dosage is essential for adrenal fate determination. It results in steroidogenic differentiation, increased cell proliferation and inhibition of apoptosis. Sf-1 targets involved in cell fate determination, proliferation and apoptosis are mostly unidentified. 


\section{References}

1. Orth, D.N. and N.J. Kovacs, The adrenal cortex, in Williams Text book of Endocrinology, 9th edition, J. Wilson, et al., Editors. 1998, WB Saunders company, Philadelphia. p. 517-664.

2. Hershkovitz, L., et al., Adrenal 20alpha-hydroxysteroid dehydrogenase in the mouse catabolizes progesterone and 11-deoxycorticosterone and is restricted to the $X$-zone. Endocrinology, 2007. 148(3): p. 976-88.

3. Zubair, M., et al., Two-step regulation of $A d 4 B P / S F-1$ gene transcription during fetal adrenal development: initiation by a Hox-Pbxl-Prepl complex and maintenance via autoregulation by Ad4BP/SF-1. Mol Cell Biol, 2006. 26(11): p. 4111-21.

4. Zubair, M., K.L. Parker, and K.I. Morohashi, Developmental links between the fetal and adult adrenal cortex revealed by lineage tracing. Mol Cell Biol, 2008. 28(23): p. 7030-7040.

5. Keegan, C.E. and G.D. Hammer, Recent insights into organogenesis of the adrenal cortex. Trends Endocrinol Metab, 2002. 13(5): p. 200-8.

6. Else, T. and G.D. Hammer, Genetic analysis of adrenal absence:agenesis and aplasia. Trends Endocrinol Metab, 2005. 16(10): p. 458-468.

7. Mesiano, S. and R.B. Jaffe, Developmental and functional biology of the primate fetal adrenal cortex. Endocr Rev, 1997. 18(3): p. 378-403.

8. Kempna, P. and C.E. Fluck, Adrenal gland development and defects. Best Pract Res Clin Endocrinol Metab, 2008. 22(1): p. 77-93.

9. Hatano, O., et al., Identical origin of adrenal cortex and gonad revealed by expression profiles of Ad4BP/SF-1. Genes Cells, 1996. 1(7): p. 663-71.

10. Heikkila, M., et al., Wnt-4 deficiency alters mouse adrenal cortex function, reducing aldosterone production. Endocrinology, 2002. 143(11): p. 4358-4365.

11. O'Shaughnessy, P.J., et al., Adrenocorticotropic hormone directly stimulates testosterone production by the fetal and neonatal mouse testis. Endocrinology, 2003. 144(8): p. 3279-3284.

12. Wang, G.M., et al., Expression of 11- $\beta$ hydroxylase in rat Leydig cells. Endocrinology, 2002. 143(2): p. 621-626.

13. $\mathrm{Hu}, \mathrm{L}$. , et al., Expression of Cyp21al and Cyp11b1 in the fetal mouse testis. Reproduction, 2007. 134(4): p. 585-91.

14. Val, P., C. Jeays-Ward, and A. Swain, Identification of a novel population of adrenallike cells in the mammalian testis. Dev Biol, 2006. 299: p. 250-256.

15. Val, P., et al., SF-1 a key player in the development and differentiation of steroidogenic tissues. Nuclear Receptor, 2003. 1(8).

16. Parker, K.L., et al., Steroidogenic factor 1: an essential mediator of endocrine development. Recent Prog Horm Res, 2002. 57: p. 19-36.

17. Luo, X., Y. Ikeda, and K.L. Parker, A cell-specific nuclear receptor is essential for adrenal and gonadal development and sexual differentiation. Cell, 1994. 77(4): p. 481-90.

18. Sadovsky, Y., et al., Mice deficient in the orphan receptor steroidogenic factor 1 lack adrenal glands and gonads but express P450 side-chain-cleavage enzyme in the placenta and have normal embryonic serum levels of corticosteroids. Proc Natl Acad Sci U S A, 1995. 92(24): p. 10939-43.

19. Ikeda, Y., et al., Developmental expression of mouse steroidogenic factor-1, an essential regulator of the steroid hydroxylases. Mol Endocrinol, 1994. 8(5): p. 654-62. 
20. Val, P., J.P. Martinez Barbera, and A. Swain, Adrenal development is initiated by Cited 2 and Wt1 through modulation of Sf-1 dosage. Development, 2007. 134(12): p. 2349-2358.

21. Crawford, P.A., Y. Sadovsky, and J. Milbrandt, Nuclear receptor steroidogenic factor 1 directs embryonic stem cells toward the steroidogenic lineage. Mol Cell Biol, 1997. 17(7): p. 3997-4006.

22. Tanaka, T., et al., Steroidogenic factor 1/adrenal 4 binding protein transforms human bone marrow mesenchymal cells into steroidogenic cells. J Mol Endocrinol, 2007. 39(5): p. 343-50.

23. Gondo, S., et al., Adipose tissue-derived and bone marrow-derived mesenchymal cells develop into different lineage of steroidogenic cells by forced expression of steroidogenic factor 1. Endocrinology, 2008. 149(9): p. 4717-25.

24. Beuschlein, F., et al., Steroidogenic factor-1 is essential for compensatory adrenal growth following unilateral adrenalectomy. Endocrinology, 2002. 143(8): p. 3122-35.

25. Bland, M.L., R.C. Fowkes, and H.A. Ingraham, Differential requirement for steroidogenic factor-1 gene dosage in adrenal development versus endocrine function. Mol Endocrinol, 2004. 18(4): p. 941-952.

26. Doghman, M., et al., Increased steroidogenic factor-1 dosage triggers adrenocortical cell proliferation and cancer. Mol Endocrinol, 2007. 21(12): p. 2968-87.

27. Doghman, M., et al., Nephroblastoma overexpressed/cysteine-rich protein 61/connective tissue growth factor/nephroblastoma overexpressed gene-3 (NOV/CCN3), a selective adrenocortical cell proapoptotic factor, is down-regulated in childhood adrenocortical tumors. J Clin Endocrinol Metab, 2007. 92(8): p. 325360 .

28. Hastie, N.D., Life, sex, and WT1 isoforms--three amino acids can make all the difference. Cell, 2001. 106(4): p. 391-4.

29. Reddy, J.C. and J.D. Licht, The WT1 Wilms' Tumor suppressor gene : How much do we really know? Biochim Biophys Acta, 1996. 1287: p. 1-28.

30. Kreidberg, J.A., et al., WT-1 is required for early kidney development. Cell, 1993. 74(4): p. 679-91.

31. Hammes, A., et al., Two splice variants of the Wilms' tumor 1 gene have distinct functions during sex determination and nephron formation. Cell, 2001. 106(3): p. 31929.

32. Wilhelm, D. and C. Englert, The Wilms tumor suppressor WT1 regulates early gonad development by activation of Sf1. Genes Dev, 2002. 16(14): p. 1839-51.

33. So, P.L. and P.S. Danielian, Cloning and expression analysis of a mouse gene related to Drosophila odd-skipped. Mech Dev, 1999. 84(1-2): p. 157-60.

34. Wang, Q., et al., Odd-skipped related (Odd1) is an essential regulator of heart and urogenital development. Dev Biol, 2005. 288: p. 582-594.

35. Birk, O.S., et al., The LIM homeobox gene Lhx9 is essential for mouse gonad formation. Nature, 2000. 403(6772): p. 909-13.

36. Miyamoto, N., et al., Defects of urogenital development in mice lacking Emx2. Development, 1997. 124(9): p. 1653-1664.

37. Tien, E.S., J.W. Davis, and P. Vandel Heuven, Identification of the CBP/p300 interacting protein CITED2 as a PPAR $\alpha$ Coregulator. J Biol Chem, 2004. 279(23): p. 24053-24063.

38. Bhattacharya, S., et al., Functional role of p35srj, a novel p300/CBP binding protein, during transactivation by HIF-1. Genes Dev, 1999. 13: p. 64-75. 
39. Bamforth, S.D., et al., Cardiac malformations, adrenal agenesis, neural crest defects and exencephaly in mice lacking Cited2, a new Tfap2 co-activator. Nat Genet, 2001. 29(4): p. 469-474.

40. Bamforth, S.D., et al., Cited2 controls left-right patterning and heart development through a Nodal-Pitx2c pathway. Nat Genet, 2004. 36(11): p. 1189-1196.

41. Bragança, J., et al., Physical and functional interactions among AP-2 transcription factors, p300/CREB-binding protein, and CITED-2. J Biol Chem, 2003. 278(18): p. 16021-16029.

42. Weninger, W.J., et al., Cited2 is required both for heart morphogenesis and establishment of the left-right axis in mouse development. Development, 2005. 132: p. 1337-1348.

43. Yin, Z., et al., The essential role of Cited2, a negative regulator for HIF1-alpha, in heart development and neurulation. Proc Natl Acad Sci U S A, 2002. 99(16): p. 10488-10493.

44. Bland, M.L., et al., Haploinsufficiency of steroidogenic factor-1 in mice disrupts adrenal development leading to an impaired stress response. Proc Natl Acad Sci U S A, 2000. 97(26): p. 14488-93.

45. Babu, P.S., et al., Interaction between Dax-1 and steroidogenic factor-1 in vivo: increased adrenal responsiveness to ACTH in the absence of Dax-1. Endocrinology, 2002. 143(2): p. 665-73.

46. Park, S.Y., et al., Nuclear Receptors Sf1 and Dax1 function cooperatively to mediate somatic cell differentiation during testis development. Development, 2005. 132(10): p. 2415-2423.

47. Fatchiyah, et al., Differential gene dosage effects of Ad4BP/SF-1 on target tissue development. Biochem Biophys Res Commun, 2006. 341(4): p. 1036-1045.

48. Nachtigal, M.W., et al., Wilms' tumor 1 and Dax-1 modulate the orphan nuclear receptor SF-1 in sex-specific gene expression. Cell, 1998. 93(3): p. 445-54.

49. Achermann, J.C., et al., A mutation in the gene encoding steroidogenic factor-1 causes $X Y$ sex reversal and adrenal failure in humans. Nat Genet, 1999. 22(2): p. 125-6.

50. Achermann, J.C., et al., Gonadal determination and adrenal development are regulated by the orphan nuclear receptor steroidogenic factor-1, in a dose-dependent manner. J Clin Endocrinol Metab, 2002. 87(4): p. 1829-33.

51. Hasegawa, T., et al., Testicular dysgensis without adrenal insufficiency in a 46, XY patient with a heterozygous inactivae mutation of steroidogenic factor-1. J Clin Endocrinol Metab, 2004. 89(12): p. 5930-5935.

52. Mallet, D., et al., Gonadal Dysgenesis without adrenal insufficiency in a 46, XY patient heterozygous for the nonsense C16X mutation: a case of SF1 haploinsufficiency. J Clin Endocrinol Metab, 2004. 89(10): p. 4829-4832.

53. Coutant, R., et al., Heterozygous mutation of steroidogenic factor-1 in 46,XY subjects may mimic partial androgen insensitivity syndrome. J Clin Endocrinol Metab, 2007. 92(8): p. 2868-73.

54. Kohler, B., et al., Five novel mutations in steroidogenic factor 1 (SF1, NR5A1) in 46,XY patients with severe underandrogenization but without adrenal insufficiency. Hum Mutat, 2008. 29(1): p. 59-64.

55. Reuter, A.L., et al., A novel mutation in the accessory DNA-binding domain of human steroidogenic factor 1 causes XY gonadal dysgenesis without adrenal insufficiency. Eur J Endocrinol, 2007. 157(2): p. 233-8.

56. Lin, L., et al., Heterozygous missense mutations in steroidogenic factor 1 (SF1/Ad4BP, NR5A1) are associated with 46,XY disorders of sex development with normal adrenal function. J Clin Endocrinol Metab, 2007. 92(3): p. 991-999. 
57. Stallings, N.R., et al., Development of a transgenic green fluorescent protein lineage marker for steroidogenic factor 1. Endocr Res, 2002. 28(4): p. 497-504.

58. Schnabel, C.A., L. Selleri, and M.L. Cleary, Pbxl is essential for adrenal development and urogenital differentiation. Genesis, 2003. 37(3): p. 123-130.

59. Lichtenauer, U.D., et al., Pre-B-cell transcription factor 1 and steroidogenic factor 1 synergistically regulate adrenocortical growth and steroidogenesis. Endocrinology, 2007. 148(2): p. 693-704.

60. Zakany, J. and D. Duboule, The role of Hox genes during vertebrate limb development. Curr Opin Genet Dev, 2007. 17(4): p. 359-66.

61. Zacchetti, G., D. Duboule, and J. Zakany, Hox gene function in vertebrate gut morphogenesis: the case of the caecum. Development, 2007. 134(22): p. 3967-73.

62. Katoh-Fukui, Y., et al., Male-to-female sex reversal in M33 mutant mice. Nature, 1998. 393: p. 688-692.

63. Katoh-Fukui, Y., et al., Mouse Polycomb M33 is required for splenic vascular and adrenal gland formation through regulating ad4BP/SF1 expression. Blood, 2005. 106(5): p. 1612-1620.

64. Quaggin, S.E., et al., The basic-helix-loop-helix protein podl is critically important for kidney and lung organogenesis. Development, 1999. 126(24): p. 5771-83.

65. Tamura, M., et al., Pod-1/Capsulin shows a sex-and stage-dependent expression pattern in the mouse gonad development and represses expression of Ad4BP/SF-1. Mech Dev, 2001. 102(1-2): p. 135-44.

66. Cui, S., et al., Disrupted gonadogenesis and male-to-female sex reversal in Pod1 knockout mice. Development, 2004. 131: p. 4095-4105.

67. Nomura, M., et al., An E box element is required for the expression of the ad4bp gene, a mammalian homologue of ftz-f1 gene, which is essential for adrenal and gonadal development. J Biol Chem, 1995. 270(13): p. 7453-61.

68. Lalli, E. and P. Sassone-Corsi, DAX-1, an unusual orphan receptor at the crossroads of steroidogenic function and sexual differentiation. Mol Endocrinol, 2003. 17(8): p. 1445-53.

69. Achermann, J.C., J.J. Meeks, and J.L. Jameson, Phenotypic spectrum of mutations in DAX-1 and SF-1. Mol Cell Endocrinol, 2001. 185(1-2): p. 17-25.

70. Ikeda, Y., et al., Steroidogenic factor 1 and Dax-1 colocalize in multiple cell lineages: potential links in endocrine development. Mol Endocrinol, 1996. 10(10): p. 1261-72.

71. Swain, A., et al., Mouse Daxl expression is consistent with a role in sex determination as well as in adrenal and hypothalamus function. Nat Genet, 1996. 12(4): p. 404-9.

72. Iyer, A.K. and E.R. McCabe, Molecular mechanisms of DAX1 action. Mol Genet Metab, 2004. 83(1-2): p. 60-73.

73. Xu, B., et al., DAX-1 and Steroid Receptor RNA Activator (SRA) Function as Transcriptional Coactivators for Steroidogenic Factor-1 in Steroidogenesis. Mol Cell Biol, 2009.

74. Yu, R.N., et al., Role of Ahch in gonadal development and gametogenesis. Nat Genet, 1998. 20(4): p. 353-7.

75. Fuerer, C., R. Nusse, and D. Ten Berge, Wnt signalling in development and disease. Max Delbruck Center for Molecular Medicine meeting on Wnt signaling in Development and Disease. EMBO Rep, 2008. 9(2): p. 134-8.

76. Kim, A.C., et al., Targeted disruption of beta-catenin in Sf1-expressing cells impairs development and maintenance of the adrenal cortex. Development, 2008. 135(15): p. 2593-602. 
77. Grigoryan, T., et al., Deciphering the function of canonical Wnt signals in development and disease: conditional loss- and gain-of-function mutations of betacatenin in mice. Genes Dev, 2008. 22(17): p. 2308-41.

78. Kim, A.C. and G.D. Hammer, Adrenocortical cells with stem/progenitor cell properties: recent advances. Mol Cell Endocrinol, 2007. 265-266: p. 10-6.

79. Tissier, F., et al., Mutations of beta-catenin in adrenocortical tumors: activation of the Wnt signaling pathway is a frequent event in both benign and malignant adrenocortical tumors. Cancer Res, 2005. 65(17): p. 7622-7.

80. Gaujoux, S., et al., Wnt/beta-catenin and 3',5'-cyclic adenosine 5'monophosphate/protein kinase A signaling pathways alterations and somatic betacatenin gene mutations in the progression of adrenocortical tumors. J Clin Endocrinol Metab, 2008. 93(10): p. 4135-40.

81. Bland, M.L., et al., Gene dosage effects of steroidogenic factor 1 (SF-1) in adrenal development and the stress. Endocr Res, 2000. 26(4): p. 515-6.

82. Figueiredo, B.C., et al., Amplification of the Steroidogenic Factor 1 (SF-1) gene in childhood adrenocortical tumors. J Clin Endocrinol Metab, 2005. 90(2): p. 615-619.

83. DiGiammarino, E.L., et al., A novel mechanism of tumorigenesis involving $p H$ dependent destabilization of a mutant p53 tetramer. Nat Struct Biol, 2001. 9(1): p. 12 16.

84. Ribeiro, R.C., et al., An inherited p53 mutation that contributes in a tissue-specific manner to pediatric adrenal cortical carcinoma. Proc Natl Acad Sci U S A, 2001. 98(16): p. 9330-9335.

85. Lefrancois-Martinez, A.M., et al., Decreased expression of cyclic adenosine monophosphate-regulated aldose reductase (AKR1B1) is associated with malignancy in human sporadic adrenocortical tumors. J Clin Endocrinol Metab, 2004. 89(6): p. 3010-3019.

86. Gummow, B.M., J.N. Winnay, and G.D. Hammer, Convergence of Wnt signaling and steroidogenic factor-1 (SF-1) on transcription of the rat inhibin alpha gene. J Biol Chem, 2003. 278(29): p. 26572-9.

87. Jordan, B.K., et al., Wnt4 overexpression disrupts normal testicular vasculature and inhibits testosterone synthesis by repressing steroidogenic factor $1 / \beta$-catenin synergy. Proc Natl Acad Sci U S A, 2003. 100(19): p. 10866-10871.

88. Mizusaki, H., et al., Dax-1 Gene Transcription is Regulated by Wnt4 in the Female Developing Gonad. Mol Endocrinol, 2003. 23: p. 23.

89. Fayard, E., J. Auwerx, and K. Schoonjans, LRH-1: an orphan nuclear receptor involved in development, metabolism and steroidogenesis. Trends Cell Biol, 2004. 14(5): p. 250-260.

90. Schoonjans, K., et al., Liver receptor homolog 1 contributes to intestinal tumor formation through effects on cell cycle and inflammation. Proc Natl Acad Sci U S A, 2005. 102(6): p. 2058-62.

91. Botrugno, O.A., et al., Synergy between LRH-1 and beta-catenin induces G1 cyclinmediated cell proliferation. Mol Cell, 2004. 15(4): p. 499-509.

92. Chassot, A.A., et al., Activation of beta-catenin signaling by Rspol controls differentiation of the mammalian ovary. Hum Mol Genet, 2008. 17(9): p. 1264-77.

93. Liu, C.F., et al., Sex-Specific Roles of \{beta\}-catenin in Mouse Gonadal Development. Hum Mol Genet, 2008.

94. Jeays-Ward, C., et al., Endothelial and steroidogenic cell migration are regulated by WNT4 in the developing mammalian gonad. Development, 2003. 130(16): p. 36633670 . 
95. King, P.J., L. Guasti, and E. Laufer, Hedgehog signalling in endocrine development and disease. J Endocrinol, 2008. 198(3): p. 439-50.

96. Yao, H.H., W. Whoriskey, and B. Capel, Desert Hedgehog/Patched 1 signaling specifies fetal Leydig cell fate in testis organogenesis. Genes Dev, 2002. 16(11): p. 1433-40.

97. Hall, J.G., et al., Congenital hypothalamic hamartoblastoma, hypopituitarism, imperforate anus and postaxial polydactyly--a new syndrome? Part I: clinical, causal, and pathogenetic considerations. Am J Med Genet, 1980. 7(1): p. 47-74.

98. Kang, S., et al., GLI3 frameshift mutations cause autosomal dominant Pallister-Hall syndrome. Nat Genet, 1997. 15(3): p. 266-8.

99. Bose, J., L. Grotewold, and U. Ruther, Pallister-Hall syndrome phenotype in mice mutant for Gli3. Hum Mol Genet, 2002. 11(9): p. 1129-35.

100. Bitgood, M.J. and A.P. McMahon, Hedgehog and Bmp genes are coexpressed at many diverse sites of cell-cell interaction in the mouse embryo. Dev Biol, 1995. 172(1): p. 126-38.

101. Katoh, Y. and M. Katoh, Hedgehog signaling, epithelial-to-mesenchymal transition and miRNA (review). Int J Mol Med, 2008. 22(3): p. 271-5.

102. Katoh, M., WNT signaling pathway and stem cell signaling network. Clin Cancer Res, 2007. 13(14): p. 4042-5.

103. Roop, D. and R. Toftgard, Hedgehog in wnterland. Nat Genet, 2008. 40(9): p. 1040-1.

104. Alvarez-Medina, R., et al., Wnt canonical pathway restricts graded Shh/Gli patterning activity through the regulation of Gli3 expression. Development, 2008. 135(2): p. 23747.

105. Cotton, L.M., M.K. O'Bryan, and B.T. Hinton, Cellular signaling by fibroblast growth factors (FGFs) and their receptors (FGFRs) in male reproduction. Endocr Rev, 2008. 29(2): p. 193-216.

106. Colvin, J.S., et al., Male-to-female sex reversal in mice lacking fibroblast growth factor 9. Cell, 2001. 104: p. 875-889.

107. Schmahl, J., et al., Fgf9 induces proliferation and nuclear localization of FGFR2 in Sertoli precursors during male sex determination. Development, 2004. 131: p. 36273636.

108. Kim, Y., et al., Fibroblast growth factor receptor 2 regulates proliferation and Sertoli differentiation during male sex determination. Proc Natl Acad Sci U S A, 2007. 104(42): p. 16558-63.

109. Basile, D.P. and M.A. Holzwarth, Basic fibroblast growth factor may mediate proliferation in the compensatory adrenal growth response. Am J Physiol, 1993. 265(6 Pt 2): p. R1253-61.

110. Basile, D.P. and M.A. Holzwarth, Basic fibroblast growth factor receptor in the rat adrenal cortex: effects of suramin and unilateral adrenalectomy on receptor numbers. Endocrinology, 1994. 134(6): p. 2482-9.

111. Holzwarth, M.A., Evidence for fibroblast growth factor mediation of compensatory adrenocortical proliferation. Endocr Res, 1995. 21(1-2): p. 115-9.

112. Ishimoto, H., et al., The periphery of the human fetal adrenal gland is a site of angiogenesis: zonal differential expression and regulation of angiogenic factors. $\mathrm{J}$ Clin Endocrinol Metab, 2008. 93(6): p. 2402-8.

113. Mesiano, S. and R.B. Jaffe, Role of growth factors in the developmental regulation of the human fetal adrenal cortex. Steroids, 1997. 62(1): p. 62-72.

114. Yaswen, L., et al., Obesity in the mouse model of pro-opiomelanocortin deficiency responds to peripheral melanocortin. Nat Med, 1999. 5(9): p. 1066-70. 
115. Coll, A.P., et al., The effects of proopiomelanocortin deficiency on murine adrenal development and responsiveness to adrenocorticotropin. Endocrinology, 2004. 145(10): p. 4721-7.

116. Challis, B.G., et al., Mice lacking pro-opiomelanocortin are sensitive to high-fat feeding but respond normally to the acute anorectic effects of peptide-YY(3-36). Proc Natl Acad Sci U S A, 2004. 101(13): p. 4695-700.

117. Looyenga, B.D. and G.D. Hammer, Genetic removal of Smad3 from inhibin-null mice attenuates tumor progression by uncoupling extracellular mitogenic signals from the cell cycle machinery. Mol Endocrinol, 2007. 21(10): p. 2440-57.

118. Looyenga, B.D. and G.D. Hammer, Origin and identity of adrenocortical tumors in inhibin knockout mice: implications for cellular plasticity in the adrenal cortex. Mol Endocrinol, 2006. 20(11): p. 2848-63. 


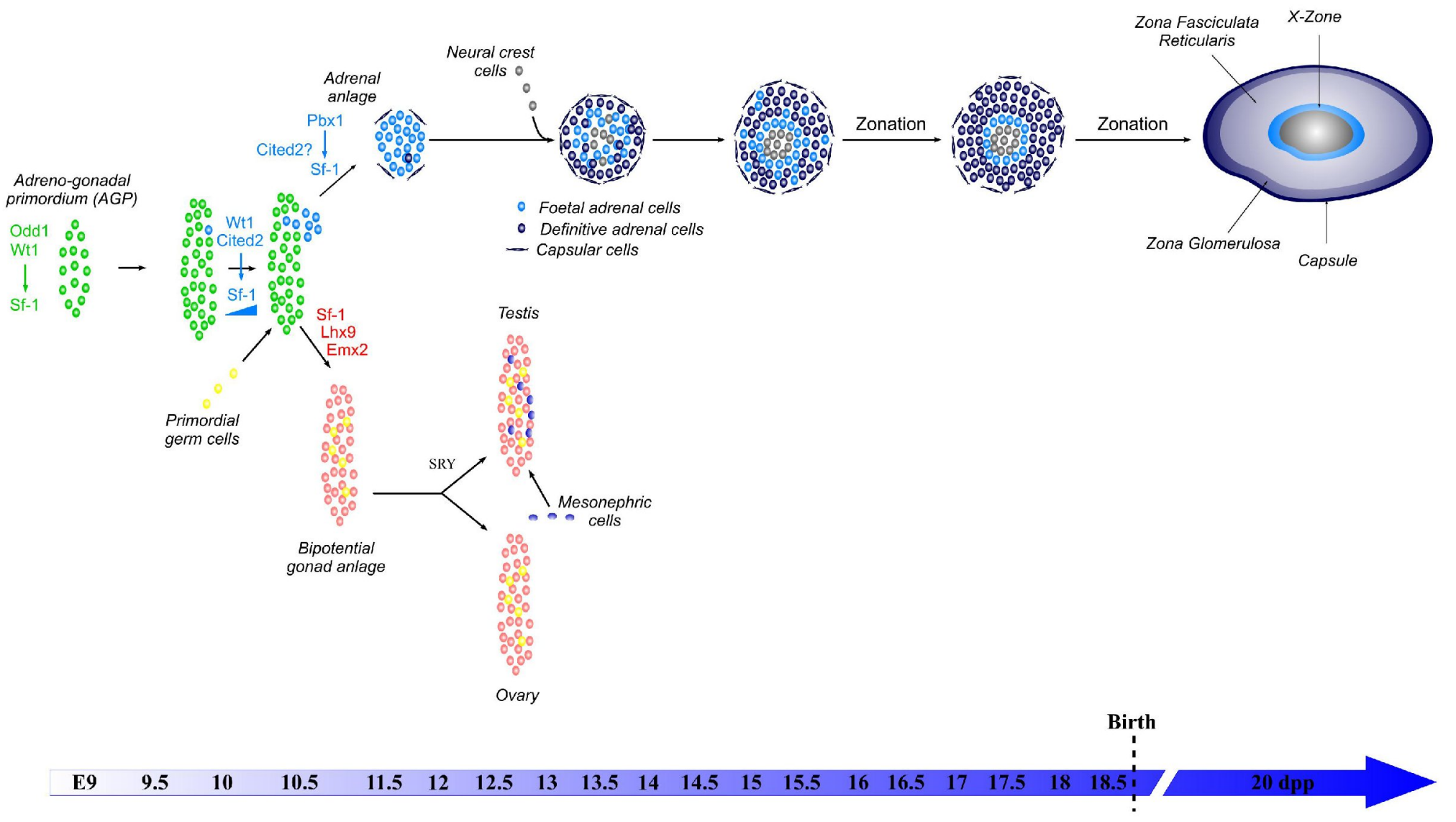

Figure 1. 


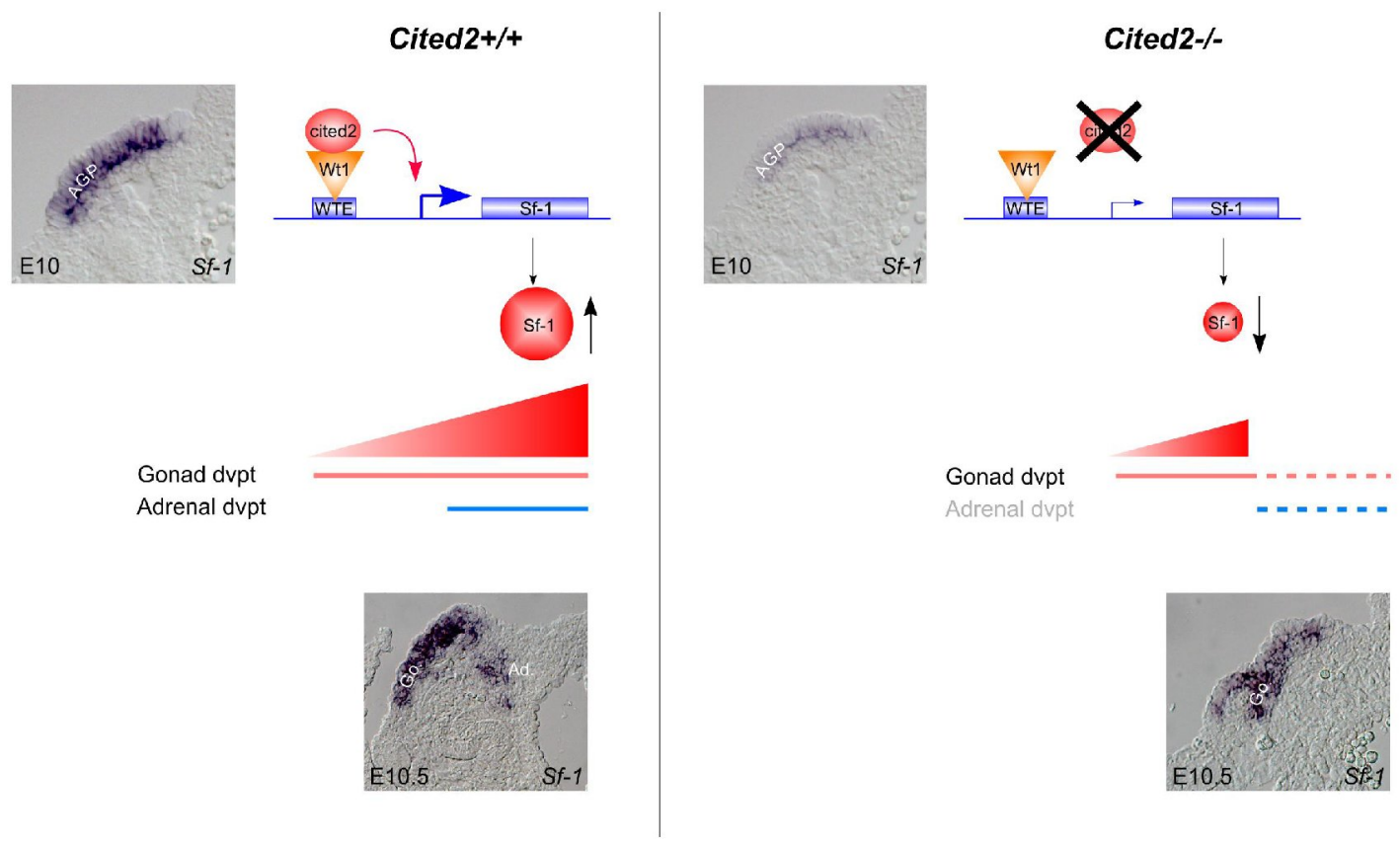

Figure 2. 


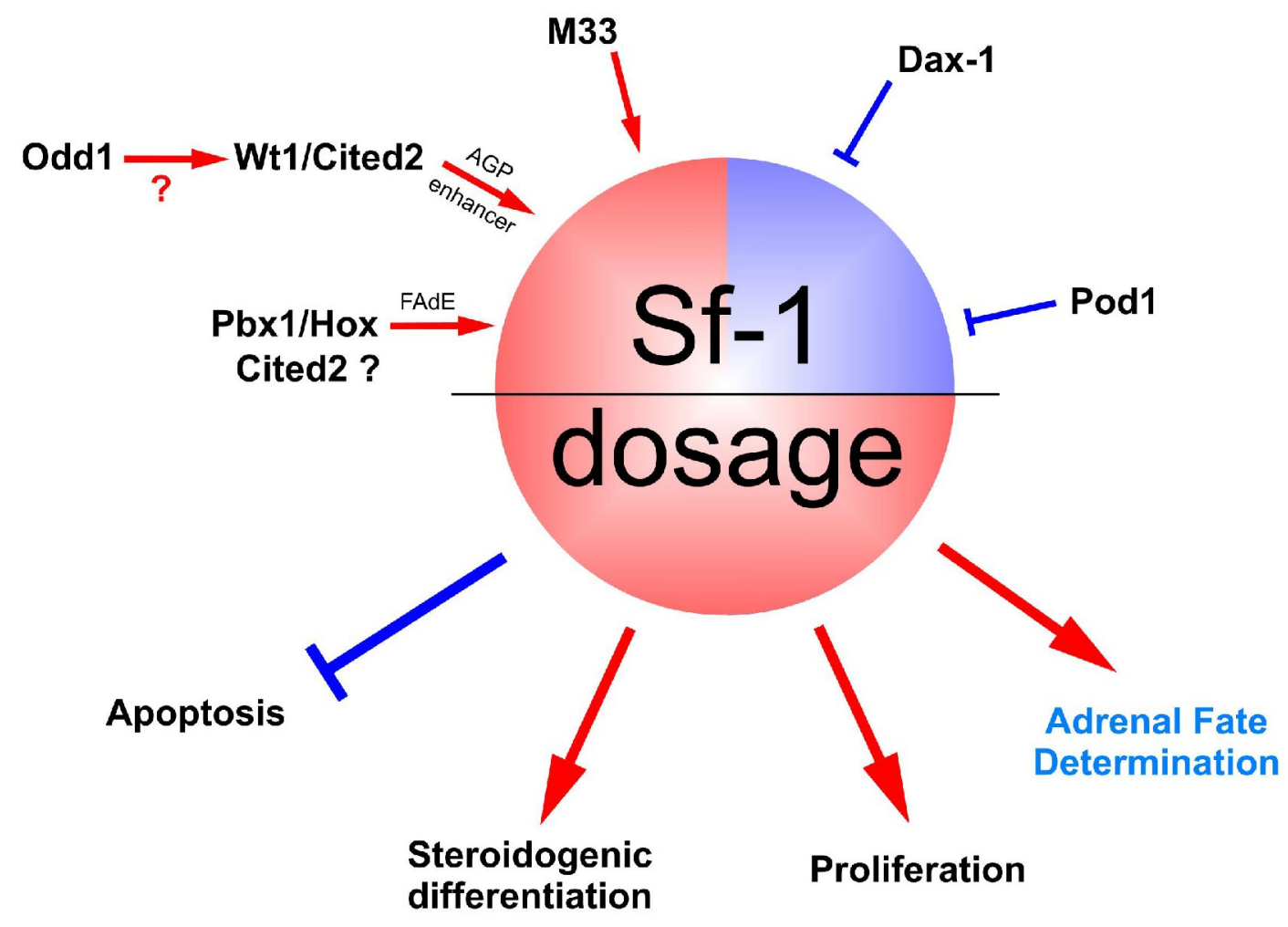

Figure 3. 